\title{
LAMINAR NATURAL CONVECTION OVER A FRUSTUM OF A CONE
}

\author{
T. Y, NA
}

Professor of Mechanical Engineering, The University of Michigan-Dearborn, Dearborn, Michigan, 48128, U.S.A.

\section{J. P. CHIOU}

Professor of Mechanical Engineering, University of Detroit, Detroit, Michigan, 48221, U.S.A.

\begin{abstract}
The problem of laminar, natural convection flow over a vertical frustum of a cone is treated in this paper. The thermal boundary condition at the wall include both the constant wall temperature and the constant wall heat flux cases. The governing differential equations are solved by a combination of quasilinearization and finitedifference methods. Numerical solutions are obtained for a range of Prandtl numbers. The solutions are found to approach to the solutions for a full cone if the flow is far downstream or the radius of the cross-section at the leading edge is very small.
\end{abstract}

\section{Introduction}

In a recent study of the effect of slenderness on the natural convection flow over a slender frustum of a cone [1,2], the authors found that the problem of natural convection flow over a frustum of a cone without transverse curvature effect (i.e., large cone angles when the boundary layer thickness is small compared with the local radius of the cone) has not been treated in the literature, even though the problem for a full cone has been treated quite extensively [3-11]. It is therefore the purpose of this paper to present an analysis of this problem (see Fig. 1) when the thermal boundary condition on the wall is constant either in the wall temperature or in the wall heat flux.

The physical model and the coordinate system are shown in Figure 1. Similar to the analysis of the natural convection flow over a slender circular cylinder by Sparrow and Gregg [12], the boundary layer is 


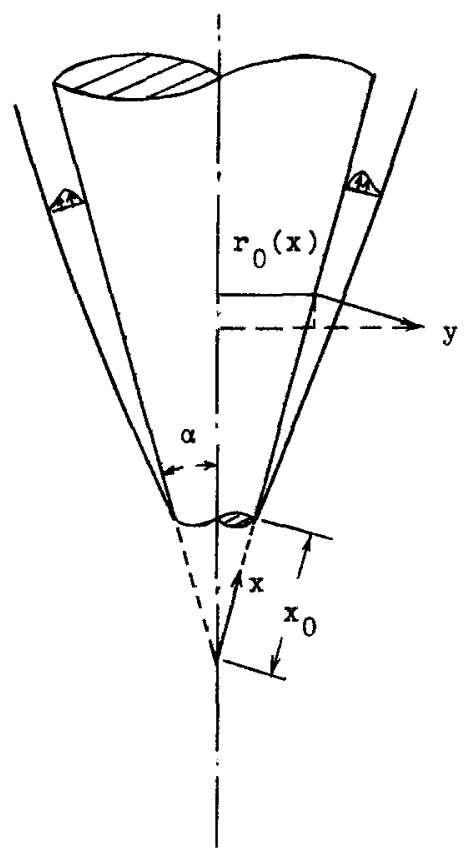

Fig. 1 The Coordinate system

assumed to develop at the leading edge $\left(x=x_{0}\right)$, which means the temperature at the circular base is assumed to be at the same temperature as the temperature of the surrounding fluid. Due to the difference in temperature between the surface and the surrounding fluid, an upward flow is created as a result of buoyancy. As in similar analyses of such problems, the boundary layer approximations are assumed to be valid. The flow is steady and fluid-property variations are assumed negligible except for the density variation necessary to create the buoyancy force.

The boundary layer equations for the problem under consideration can therefore be written in dimensionless quantities as:

$$
\begin{aligned}
\frac{\partial\left(\bar{r}_{0} \bar{u}\right)}{\partial \bar{x}}+\frac{\partial\left(\bar{r}_{0} \bar{v}\right)}{\partial \bar{y}}=0 \\
\bar{u} \frac{\partial \bar{u}}{\partial \bar{x}}+\bar{v} \frac{\partial \bar{u}}{\partial \bar{y}}=\frac{\partial^{2} \bar{u}}{\partial \bar{y}^{2}}+\theta \\
\bar{u} \frac{\partial \theta}{\partial \bar{x}}+\bar{v} \frac{\partial \theta}{\partial \bar{y}}=\frac{1}{\operatorname{Pr}} \frac{\partial^{2} \theta}{\partial \bar{y}^{2}}
\end{aligned}
$$


The dimensionless quantities in Equations (1), (2) and (3) are related to their corresponding physical variables through the following definitions:

$$
\begin{aligned}
& \bar{x}=\frac{x-x_{0}}{L} ; \quad \bar{y}=\frac{y}{L} \sqrt{\operatorname{Re}_{L}} ; \quad \bar{u}=\frac{u}{u_{c}} ; \quad \bar{v}=\frac{v}{u_{c}} \sqrt{\operatorname{Re}_{L}} ; \\
& \bar{r}_{0}=\frac{r_{o}(x)}{L} \sqrt{\operatorname{Re}_{L}} ; \quad \theta=\frac{T-T_{\infty}}{\theta_{r}} ; \quad \bar{r}=\frac{r}{L} \sqrt{\operatorname{Re}_{L}}
\end{aligned}
$$

and

$$
\operatorname{Re}_{L}=\frac{u_{c} L}{u}
$$

where, for the case of constant wall temperature,

$$
\begin{aligned}
u_{c} & =\left[g_{e} \beta \operatorname{Cos} \alpha\left(T_{w}-T_{\infty}\right) L\right]^{1 / 2} \\
\theta_{r} & =T_{w}-T_{\infty}
\end{aligned}
$$

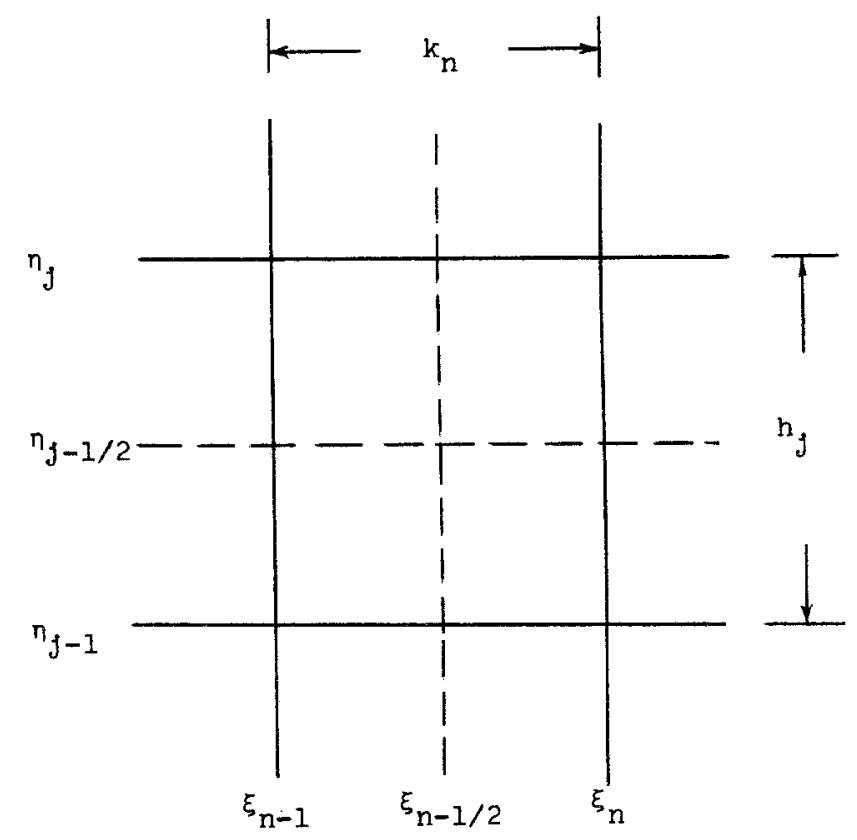

Fig. 2 A typical grid point 
and, for the case of constant wall heat flux,

$$
\begin{aligned}
& u_{c}=\left[g_{e} \beta \operatorname{Cos} \alpha\left(\frac{q_{0} L}{k}\right)(L \nu)^{1 / 2}\right]^{2 / 5} \\
& \theta_{r}=\frac{q_{0} L}{k \sqrt{\operatorname{Re}_{L}}}
\end{aligned}
$$

If the characteristic length, $L$, is chosen as the distance $x_{0}$ (see Fig. 1), then the Reynolds number can be expressed in terms of the Grashof number. For the case of constant wall temperature, we have:

$$
\operatorname{Re}_{L}=\frac{u_{c} x_{0}}{\nu}=\left\{\frac{g_{e} \beta \operatorname{Cos} \alpha\left(T_{w}-T_{\infty}\right) x_{0}^{3}}{\nu^{2}}\right\}^{1 / 2}=G r_{x_{0}}^{1 / 2}
$$

and, for the case of constant wall heat flux, we have

$$
\operatorname{Re}_{L}=\frac{u_{c} x_{0}}{\nu}=\left\{\frac{g_{e} \beta \operatorname{Cos} \alpha\left(q_{0} x_{0} / k\right) x_{0}^{3}}{\nu^{2}}\right\}^{2 / 5}=G r_{x_{0}}^{2 / 5}
$$

where $G r_{x_{0}}$ is the Grashof number based on $x_{0}$.

The boundary conditions are:

$$
\begin{array}{ll}
\bar{y}=0: & \bar{u}=0 ; \quad \bar{v}=0 \\
\theta & =1 \text { (for the case of constant wall temperature) } \\
& \frac{\partial \theta}{\partial \bar{y}}=-1 \text { (for the case of constant wall heat flux) } \\
\bar{y}=\infty: \quad \bar{u}=0 ; \quad \theta=0
\end{array}
$$

We will now seek solutions of the differential equations for the two cases. The numerical method used in this paper is identical to the one used in reference 15 and will therefore not be discussed here. A very brief outline is given in the appendix.

\section{Numerical Solutions}

Case 1. Constant Wall Temperature

Let us define the stream function as

$$
\bar{r}_{0} \bar{u}=\frac{\partial \bar{\psi}}{\partial \bar{y}} ; \quad \bar{r}_{0} \bar{v}=-\frac{\partial \psi}{\partial \bar{x}}
$$


and the transformation

$$
\begin{gathered}
\xi=\bar{x}, \quad \eta=\frac{\bar{y}}{\bar{x}^{1 / 4}} \\
f(\xi, \eta)=\frac{\bar{\psi}}{\bar{x}^{3 / 4} \bar{r}_{0}}, \quad g(\xi, \eta)=\theta
\end{gathered}
$$

where, for cones, $r_{0}$ is related to $x$ by

$$
r_{0}=x \operatorname{Sin} \alpha
$$

Equations (1), (2) and (3) then become:

$$
\begin{array}{r}
f^{\prime \prime \prime}+\left(R+\frac{3}{4}\right) f f^{\prime \prime}-\frac{1}{2}\left(f^{\prime}\right)^{2}+g=\xi\left(f^{\prime} \frac{\partial f^{\prime}}{\partial \xi}-f^{\prime \prime} \frac{\partial f}{\partial \xi}\right) \\
g^{\prime \prime}+\left(R+\frac{3}{4}\right) \operatorname{Pr} f g^{\prime}=\xi\left(f^{\prime} \frac{\partial g}{\partial \xi}-g^{\prime} \frac{\partial f}{\partial \xi}\right)
\end{array}
$$

subject to the boundary conditions:

$$
\begin{aligned}
f(\xi, 0) & =f^{\prime}(\xi, 0)=0 ; \quad g(\xi, 0)=1 \\
f^{\prime}(\xi, \infty) & =0 ; \quad g(\xi, \infty)=0
\end{aligned}
$$

where

$$
R=\frac{\xi}{1+\xi}
$$

At $\xi=0$, the ratio is zero and Equations (15) and (16) are reduced to:

$$
\begin{aligned}
& f^{\prime \prime \prime}+\frac{3}{4} f f^{\prime \prime}-\frac{1}{2}\left(f^{\prime}\right)^{2}+g=0 \\
& g^{\prime \prime}+\frac{3}{4} \operatorname{Pr} f g^{\prime}=0
\end{aligned}
$$

subject the boundary conditions:

$$
\begin{aligned}
& f(0)=f^{\prime}(0)=0 ; \quad g(0)=1 \\
& f^{\prime}(\infty)=0 ; \quad g(\infty)=0
\end{aligned}
$$

On the other hand, as $\xi$ becomes very large, the ratio $R$ approaches to 1 and the solutions are expected to approach to the similarity solutions of the natural convection flow over a full cone [3], namely,

$$
\begin{aligned}
& f^{\prime \prime \prime}+\frac{7}{4} f f^{\prime \prime}-\frac{1}{2}\left(f^{\prime}\right)^{2}+g=0 \\
& g^{\prime \prime}+\frac{7}{4} \operatorname{Pr} f g^{\prime}=0
\end{aligned}
$$


subject to the boundary conditions:

$$
\begin{aligned}
& f(0)=f^{\prime}(0)=0 ; \quad g(0)=1 \\
& f^{\prime}(\infty)=0 ; \quad g(\infty)=0
\end{aligned}
$$

Equations (20) and (21) can also be obtained by setting $R=1$ into the left-hand side of equations (15) and (16) and equating the right-hand sides of these equations to zero. Actual computations of the solutions of equations (15) and (16) bears out this prediction.

The heat transfer rate is given by:

$$
\begin{aligned}
q_{w} & =-k\left(\frac{\partial T}{\partial y}\right)_{y=0} \\
& =\frac{k\left(T_{w}-T_{\infty}\right)}{x_{0}} G r_{x_{0}}^{1 / 4} \xi^{-1 / 4} g^{\prime}(\xi, 0) \\
& =h_{x}\left(T_{w}-T_{\infty}\right)
\end{aligned}
$$

The Nusselt number is therefore:

$$
\begin{aligned}
N u_{x^{*}} & =\frac{h_{x} x^{*}}{k} \\
& =G r_{x_{0}}^{1 / 4} \xi^{3 / 4}\left[-g^{\prime}(\xi, 0)\right] \\
& =G r_{x^{*}}^{1 / 4}\left[-g^{\prime}(\xi, 0)\right]
\end{aligned}
$$

where $x^{*}=x-x_{0}$, and

$$
G r_{x^{*}}=\frac{g_{e} \beta \operatorname{Cos} \alpha\left(T_{w}-T_{\infty}\right) x^{* 3}}{\nu^{2}}
$$

First, $\left[-g^{\prime}(\xi, o)\right]$ are tabulated in Table 1 for the two limiting cases

TABLE 1. $\left[-g^{\prime}(0,0)\right]$ and $\left[-g^{\prime}(\infty, 0)\right]$ for various Prandtl numbers

\begin{tabular}{rll}
\hline & $\begin{array}{l}{\left[-g^{\prime}(0,0)\right]} \\
\text { solutions of }(18) \\
\text { and (19) }\end{array}$ & $\begin{array}{l}{\left[-g^{\prime}(\infty, 0)\right]} \\
\text { solutions of }(20) \\
\text { and (21) }\end{array}$ \\
\hline 0.01 & 0.05742 & 0.07493 \\
0.70 & 0.35320 & 0.45101 \\
1.00 & 0.40110 & 0.51039 \\
10.00 & 0.82690 & 1.03397 \\
100.00 & 1.54930 & 1.92197 \\
\hline
\end{tabular}


TABLE 2. $\left[-g^{\prime}(\xi, 0)\right]$ for various Prandtl numbers

\begin{tabular}{r|ccccc}
\hline \multirow{2}{*}{$\xi$} & \multicolumn{5}{|c}{$\left[-g^{\prime}(\xi, 0)\right]$} \\
\cline { 2 - 6 }$\xi$ & $\operatorname{Pr}=0.01$ & $\operatorname{Pr}=0.70$ & $\operatorname{Pr}=1.0$ & $\operatorname{Pr}=10$. & $\operatorname{Pr}=100$. \\
\hline 0.00 & 0.05742 & 0.35320 & 0.40110 & 0.82690 & 1.54930 \\
0.25 & 0.06135 & 0.37202 & 0.42185 & 0.86336 & 1.61215 \\
0.75 & 0.06536 & 0.39204 & 0.44404 & 0.90293 & 1.68088 \\
1.75 & 0.06895 & 0.41366 & 0.46830 & 0.95023 & 1.76690 \\
3.75 & 0.07148 & 0.42893 & 0.48525 & 0.98229 & 1.82496 \\
7.75 & 0.07307 & 0.43958 & 0.49749 & 1.00866 & 1.87572 \\
15.75 & 0.07397 & 0.44462 & 0.50312 & 1.01835 & 1.89189 \\
31.75 & 0.07444 & 0.44822 & 0.50726 & 1.02848 & 1.91286 \\
63.75 & 0.07468 & 0.44916 & 0.50827 & 1.02889 & 1.92150 \\
127.75 & 0.07481 & 0.45052 & 0.50987 & 1.03369 & 1.92189 \\
$\infty$ & 0.07493 & 0.45101 & 0.51039 & 1.03397 & 1.92197 \\
\hline
\end{tabular}

defined by equations (18)-(21). The complete solutions (from $\xi=0$ to $\xi=\infty)$ are tabulated in Table 2 and Fig. 3 for the same Prandtl numbers. It is seen that the solutions start at $\xi=0$ from solutions of equations (18) and (19) and approach to the similarity solutions of natural convection over a full cone, Eqs. (20) and (21), as $\xi$ becomes very large.

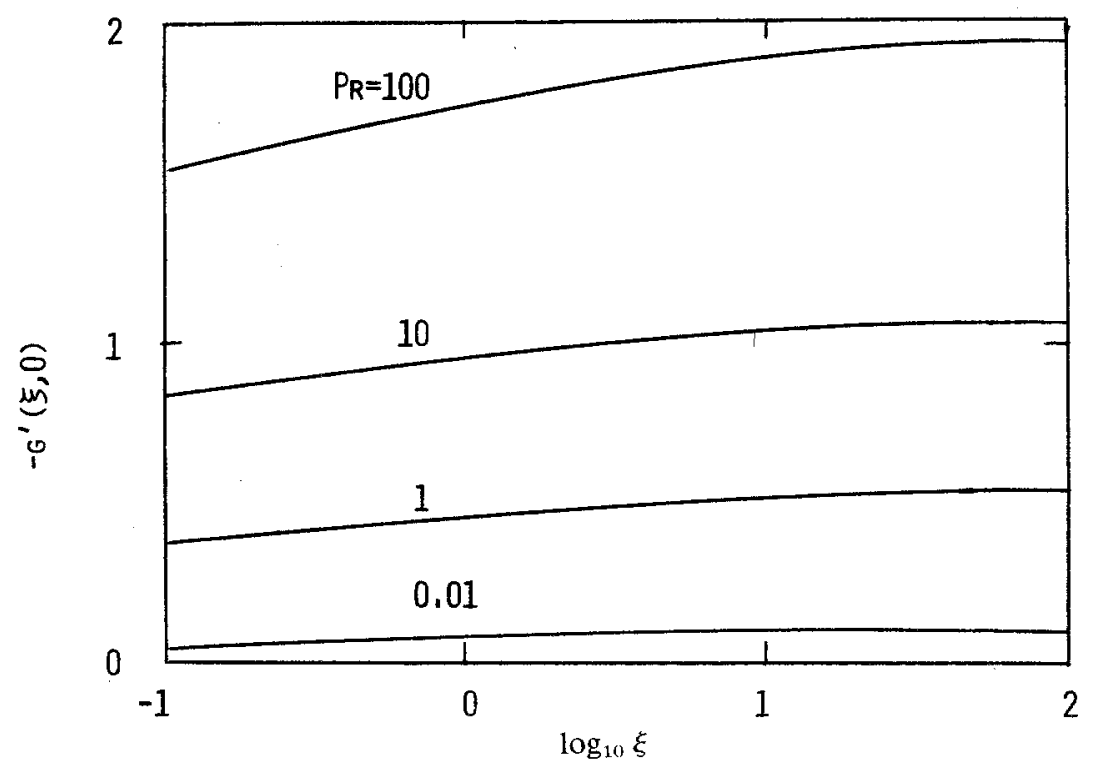

Fig. $3-G^{\prime}(\xi, 0)-v s-\xi$ for various Prandtl numbers 
Case 2. Constant Wall Heat Flux

Introducing the stream function defined in Eq. (12) and the transformation defined by:

$$
\begin{aligned}
& \xi=\bar{x}, \quad \eta=\frac{\bar{y}}{\bar{x}^{1 / 5}} \\
& f(\xi, \eta)=\frac{\psi}{\bar{x}^{4 / 5} \bar{r}_{0}}, \quad g(\xi, \eta)=\frac{\theta}{\bar{x}^{1 / 5}}
\end{aligned}
$$

Equations (1), (2) and (3) become:

$$
\begin{aligned}
& f^{\prime \prime \prime}+\left(R+\frac{4}{5}\right) f f^{\prime \prime}-\frac{3}{5}\left(f^{\prime}\right)^{2}+g=\xi\left(f^{\prime} \frac{\partial f^{\prime}}{\partial \xi}-f^{\prime \prime} \frac{\partial f}{\partial \xi}\right) \\
& g^{\prime \prime}+\left(R+\frac{4}{5}\right) \operatorname{Pr} f g^{\prime}-\frac{1}{5} \operatorname{Pr} f^{\prime} g=\xi \operatorname{Pr}\left(f^{\prime} \frac{\partial g}{\partial \xi}-g^{\prime} \frac{\partial f}{\partial \xi}\right)
\end{aligned}
$$

subject to the boundary conditions:

$$
\begin{aligned}
& f(\xi, 0)=f^{\prime}(\xi, 0)=0, \quad g^{\prime}(\xi, 0)=-1 \\
& f^{\prime}(\xi, \infty)=0, \quad g(\xi, \infty)=0
\end{aligned}
$$

where $R$ is defined in equation (17).

At $\xi=0$ (where $R=0$ ), equations (26) and (27) become:

$$
\begin{array}{r}
f^{\prime \prime \prime}+\frac{4}{5} f f^{\prime \prime}-\frac{3}{5}\left(f^{\prime}\right)^{2}+g=0 \\
g^{\prime \prime}+\frac{4}{5} \operatorname{Pr} f g^{\prime}-\frac{1}{5} \operatorname{Pr} f^{\prime} g=0
\end{array}
$$

subject to the boundary conditions:

$$
\begin{aligned}
& f(0)=f^{\prime}(0)=0, \quad g^{\prime}(0)=-1 \\
& f^{\prime}(\infty)=0, \quad g(\infty)=0
\end{aligned}
$$

As $\xi$ becomes large, the ratio $\mathrm{R}$ approaches to 1 and the right-hand sides of equations (26) and (27) become negligible. Equations (26) and (27) then become:

$$
\begin{array}{r}
f^{\prime \prime \prime}+\frac{9}{5} f f^{\prime \prime}-\frac{3}{5}\left(f^{\prime}\right)^{2}+g=0 \\
g^{\prime \prime}+\frac{9}{5} \operatorname{Pr} f g^{\prime}-\frac{1}{5} \operatorname{Pr} f^{\prime} g=0
\end{array}
$$

subject to the boundary conditions:

$$
\begin{aligned}
& f(0)=f^{\prime}(0)=0, \quad g^{\prime}(0)=-1 \\
& f^{\prime}(\infty)=0, \quad g(\infty)=0
\end{aligned}
$$


TABLE 3. $g(0,0)$ and $g(\infty, 0)$ for various Prandtl numbers

\begin{tabular}{rll} 
Pr & $\begin{array}{l}G(0,0), \text { solutions of } \\
(28) \text { and }(29)\end{array}$ & $\begin{array}{l}g(\infty, 0), \text { solutions of } \\
(30) \text { and }(31)\end{array}$ \\
\hline 0.1 & 3.7952 & 3.2781 \\
1.0 & 1.8729 & 1.6329 \\
10.0 & 1.0589 & 0.9336 \\
100.0 & 0.6425 & 0.5738 \\
\hline
\end{tabular}

Equations (30) and (31) are the similarity solutions for flows over a full cone [8]. The solutions of equations (26) and (27) are expected to change from the solutions of equations (28) and (29) to the solutions of equations (30) and (31) as $\xi$ is increased from zero to infinity.

For the case of specified constant wall heat flux, the wall temperature is the physical quantity sought. In terms of the transformed variables, the wall temperature is given by:

$$
T_{w}(x)-T_{\infty}=\frac{\left(q_{0} x_{0} / k\right) \xi^{1 / 5}}{\left\{\frac{g_{e} \beta \operatorname{Cos} \alpha\left(q_{0} x_{0} / k\right) x_{0}^{3}}{\nu^{2}}\right\}^{1 / 5}} g(\xi, 0)
$$

In order to see the role of $x_{0}$ as $x_{0}$ approaches to zero, we write

TABLE 4. $g(\xi, 0)$ for various Prandtl numbers

\begin{tabular}{r|cccc}
\hline \multirow{2}{*}{$\xi$} & \multicolumn{4}{|c}{$g(\xi, 0)$} \\
\cline { 2 - 5 }$\xi$ & $\operatorname{Pr}=0.1$ & $\operatorname{Pr}=1.0$ & $\operatorname{Pr}=10$. & $\operatorname{Pr}=100$. \\
\hline 0.00 & 3.7952 & 1.8729 & 1.0589 & 0.6425 \\
0.25 & 3.6970 & 1.8282 & 1.0363 & 0.6294 \\
0.75 & 3.5954 & 1.7841 & 1.0142 & 0.6168 \\
1.75 & 3.4876 & 1.7325 & 0.9855 & 0.5995 \\
3.75 & 3.4016 & 1.6930 & 0.9642 & 0.5870 \\
7.75 & 3.3454 & 1.6649 & 0.4473 & 0.5764 \\
15.75 & 3.3128 & 1.6498 & 0.9393 & 0.5718 \\
31.75 & 3.2950 & 1.6406 & 0.9333 & 0.5678 \\
63.75 & 3.2864 & 1.6368 & 0.9317 & 0.5671 \\
127.75 & 3.2813 & 1.6339 & 0.9295 & 0.5654 \\
$\infty$ & 3.2781 & 1.6329 & 0.9336 & 0.5738 \\
\hline
\end{tabular}




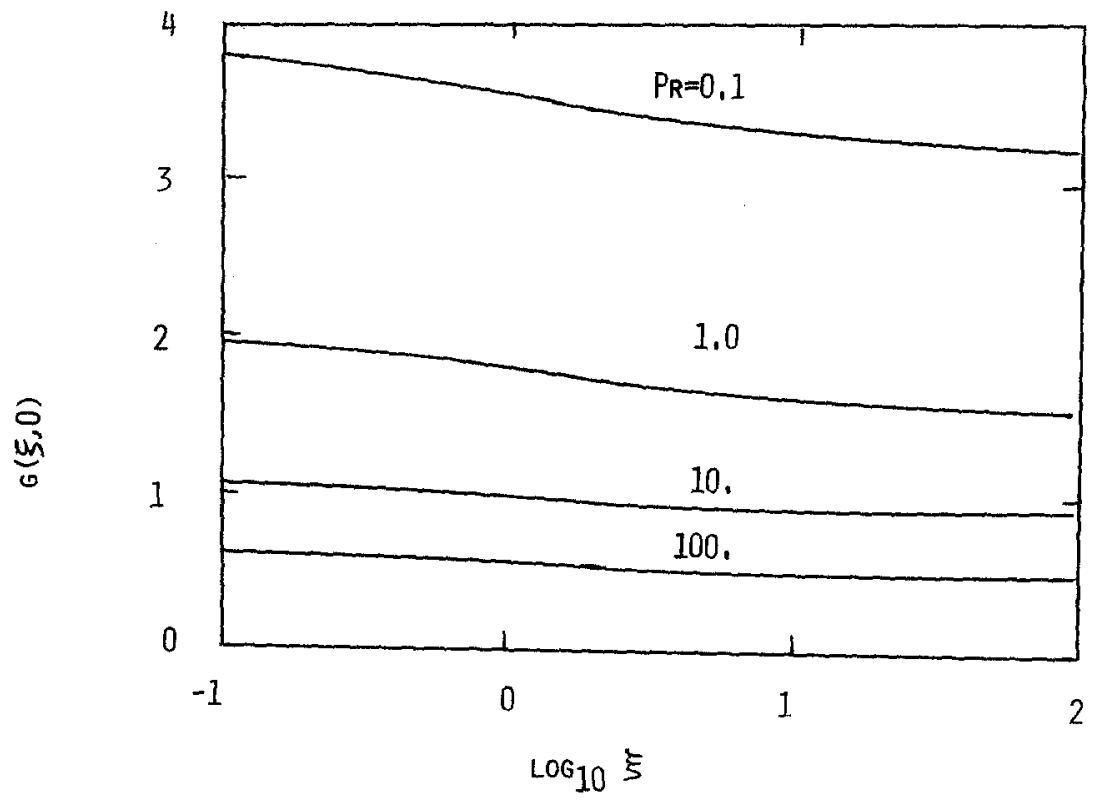

Fig. $4 G(\xi, 0)-v s-\xi$ for various Prandtl numbers

equation (32) as:

$$
T_{w}(x)-T_{\infty}=\frac{q_{0}\left(x-x_{0}\right) / k}{\left\{\frac{g_{e} \beta \operatorname{Cos} \alpha\left[q_{0}\left(x-x_{0}\right) / k\right]\left(x-x_{0}\right)^{3}}{\nu^{2}}\right\}^{1 / 5}} g(\xi, 0)
$$

First, $g(\xi, 0)$ are tabulated in Table 3 for the two limiting cases defined in equations (28)-(31). The complete solutions (from $\xi=0$ to $\xi=\infty)$ are tabulated in Table 4 and Figure 4 for the same Prandtl numbers. It is seen that the solutions start at $\xi=0$ from solutions of equations (28) and (29) and approach to the similarity solutions of natural convection over a full cone, equations (30) and (31), as $\xi$ becomes very large.

\section{Concluding Remarks}

Based on the analyses above and the numerical solutions summarized in Tables 2 and 4 and Figures 3 and 4, the following are observed:

1. For both cases, the governing differential equations are nonsimilar from $x=0$ to approximately $x=100 x_{0}$. This means solu- 
tions based on a full cone is in error in calculating either the wall heat flux or the wall temperature. Instead, equations (23) or (33) and the numerical solutions should be used. This justifies the need for the present analysis.

2. From the definition of $\xi$, namely, $\xi=\left(x-x_{0}\right) / x_{0}$, a large value of $\xi$ implies either a large $x$ (i.e., the flow far downstream) or a small $x_{0}$. In either case, the flow is expected to approach to the flow over a full cone. The numerical solutions tabulated in Tables 2 and 4 (also in Fig. 3 and Fig. 4) demonstrate clearly this point. Also, equations (23) and (33) are in such forms that when $x_{0}$ is set to zero, the expressions are reduced to those of the case of a full cone.

For the natural convection of a given fluid over a given frustum of a cone, the physical properties of the fluid, $x_{0}$ and $\alpha$ are given quantities. If, in addition, the wall temperature and the fluid temperature are both given, equation (23) gives the local heat transfer coefficient as a function of the location $\left(x-x_{0}\right)$ along the surface of the cone. On the other hand, if the wall heat flux is the known quantity, equation (33) gives the wall temperature as a function of the location $\left(x-x_{0}\right)$ along the surface of the cone.

\section{Appendix Method of solution}

To solve equations (15) and (16) [or, equations (26) and (27)], they are first written as a first-order system. The derivatives are then approximated by centered-difference gradients and averages centered at the mid-points of the net rectangles defined by:

$$
\begin{aligned}
& \xi_{0}=0, \quad \xi_{n}=\xi_{n-1}+k_{n}, \quad n=1,2, \ldots, N \\
& \eta_{0}=0, \quad \eta_{j}=\eta_{j-1}+h_{j}, \quad j=1,2, \ldots, J ; \quad \eta_{J}=\eta_{\infty}
\end{aligned}
$$

as shown in figure 2 . A non-uniform grid $h_{j}$ defined by:

$$
h_{j}=K h_{j-1}
$$

where the ratio of adjacent intervals, $K$, is a constant. The distance from the surface to the $j$ th station is then given by:

$$
\eta_{j}=h_{j} \frac{K^{j}-1}{K-1}, \quad j=1,2, \ldots, J
$$


The $\xi$-direction grid $k_{n}$ is arbitrary. Linearization is achieved by the method of quasilinearization and the resulting system of algebraic equations are then solved by a block-tridiagonal factorization technique described in reference 15 . The scheme is unconditionally stable and second-order accurate. The reader is referred to references 13 and 14 for extensive discussions of this method and its application to viscous flow problems.

\section{Nomenclature}

$f \quad$ dependent variable, defined in equation (13) or (25)

$g$ dependent variable, defined in equation (13) or (25)

$g_{e} \quad$ gravitational acceleration

$h \quad$ heat transfer coefficient, or $\eta$-grid

$k \quad$ heat conductivity, or $\xi$-grid

$L \quad$ characteristic length

$\mathrm{Nu}$ Nusselt number

Pr Prandtl number

$q$ heat flux

$r$ radial distance from the axis of the cone

$r_{0} \quad$ radius of the cone

Re Reynolds number

$T$ temperature

$u, v$ velocity components in the $x$ - and $y$-directions

$x, y$ rectangular coordinates

Greek Letters

$\theta$ dimensionless temperature, defined in equation (4)

$\beta \quad$ bulk modulus

$\alpha \quad$ cone angle

$\nu$ dynamic viscosity

$\psi \quad$ stream function

$\xi, \eta$ independent variables, defined in equation (13) or equation (25)

\section{Subscripts}

w condition at the surface

$\infty$ condition far from the surface

$r \quad$ reference condition

$o \quad$ wall condition

\section{References}

1. Na TY, JP Chiou, "Laminar Natural Convection Over a Slender Vertical Frustum of a Cone", Wärmeund-Stoffübertragung 12 (1979) pp 83-87. 
2. Na TY, J P Chiou, "Laminar Natural Convection Over A Slender Vertical Frustum of a Cone with Constant Wall Heat Flux", to appear in Wärme-und-Stoffübertragung (1979).

3. Merk EJ, JA Prins, "Thermal Convection in Laminar Boundary Layer", Appl Sci Res 4A (1953) pp 11-24, 195-206.

4. Hering RG, RJ Grosh, "Laminar Free Convection from a Non-isothermal Cone", Int'1 J of Heat and Mass Transfer 5 (1962) 1059-1067.

5. Hering RG, "Laminar Free Convection From a Non-Isothermal Cone at Low Prandtl Numbers", Int'1 J of Heat and Mass Transfer 8 (1965) pp 1333-1337.

6. Sparrow EM, LDF Guinle, "Deviation From Classical Free Convection Boundary Layer Theory at Low-Prandtl Numbers", Int'l J of Heat and Mass Transfer 11 (1968) pp 1403-1415.

7. Roy S, "Free Convection from a Vertical Cone at High Prandtl Numbers", J of Heat Transfer, Trans ASME 96 (1974) pp 115-117.

8. Lin FN, "Laminar Free Convection From a Vertical Cone With Uniform Surface Heat Flux", Letters in Heat and Mass Transfer 3 (1976) pp 49-58.

9. Kuiken HK, "Axisystemmetric Free Convection Boundary Layer Flow Past Slender Bodies", Int'l J of Heat and Mass Transfer 11 (1968) pp 1141-1153.

10. Stewart WE, "Asymptotic Calculation of Free Convection in Laminar Three Dimensional Systems", Int'l J of Heat and Mass Transfer 14 (1971) pp 1013-1032.

11. Oosthuizen PH, E Donaldson, "Free Convection Heat Transfer From Vertical Cones", J of Heat Transfer, Trans ASME 94 C3 (1972) pp 330-331.

12. Sparrow EM, JL Gregg, "Laminar Free Convection Heat Transfer From the Outer Surface of a Vertical Circular Cylinder", Trans ASME 78 (1956) pp 1823-1829.

13. Na TY, Computational Methods in Engineering Boundary Value Problems, Academic Press, Inc (1979).

14. Keller HB, "Numerical Methods in Boundary Layer Theory", Annual Review of Fluid Mechanies, 10 (1978) pp 417-433.

15. Na, TY, "Numerical Solution of Natural Convection Flow Past a Non-Isotherma1 Vertical Flat Plate", Appl Sci Res 33 (1978) pp 519-543. 